\title{
Curvature of the QCD critical line with $2+1$ HISQ fermions
}

\section{Leonardo Cosmai*}

INFN - Sezione di Bari, I-70126 Bari, Italy

E-mail: leonardo.cosmai@ba.infn.it

\section{Paolo Cea}

Dipartimento di Fisica dell'Università di Bari, I-70126 Bari, Italy

and INFN - Sezione di Bari, I-70126 Bari, Italy

E-mail: paolo.cea@ba.infn.it

\section{Alessandro Papa}

Dipartimento di Fisica, Università della Calabria, and INFN - Gruppo Collegato di Cosenza, I-87036 Rende, Italy

E-mail: papa@cs.infn.it

\begin{abstract}
We present results on the curvature of the critical line of QCD with 2+1 HISQ fermions at nonzero temperature and quark density obtained by analytic continuation from imaginary chemical potentials. Monte Carlo simulations are performed by means of the MILC code, suitably modified to include a nonzero imaginary baryon chemical potential. We set the chemical potential at the same value for the three quark species and work on the line of constant physics with a light to strange mass ratio of $1 / 20$ as determined in Ref. [1].
\end{abstract}

The 32nd International Symposium on Lattice Field Theory 23-28 June, 2014

Columbia University New York, NY

\footnotetext{
*Speaker.
} 


\section{Introduction}

Quantum Chromodynamics (QCD) is the theory underlying strong interactions and can be used to account for the different phases of strongly interacting matter under usual or unusual (extreme) conditions. A transition or rapid crossover is thought to exist from a low-temperature hadronic phase to a high-temperature Quark-Gluon Plasma (QGP) phase; the line separating these two phases in the temperature - baryon density plane is called the QCD (pseudo)critical line. The location of this line and the nature of the transition across it has many important theoretical and phenomenological implications, which go from the physics of the early Universe (high $T$ - low baryon density region of the phase diagram), to the physics of the interior of some compact astrophysical objects (corresponding to the low $T$ - high density region). Various experiments have been devised or have been planned in order to study this transition via heavy-ion collisions at ultrarelativistic energies. Depending on the beam energy, different conditions of temperature and baryon density can be realized in the fireball produced after the collision, such that the QGP phase appears as a transient state, before the system freezes out and partons recombine into hadrons. For a given collision energy, the particle yields are found to be well described by a thermal-statistical model assuming approximate chemical equilibrium, as realized at the chemical freeze-out point, in terms of only two parameters, the freeze-out temperature $T$ and the baryon chemical potential $\mu_{B}$. The set of freeze-out parameters determined in experiments with different collision energies lie on a curve in the $\left(T, \mu_{B}\right)$-plane, extending up to $\mu_{B} \lesssim 800 \mathrm{MeV}$ (see Fig. 1 of Ref. [2], or Ref. [3] for a recent re-analysis of experimental data). Chemical freeze-out is reached as the fireball cools down, subsequently to re-hadronization. A reasonable guess is that chemical freeze-out is reached shortly after hadronization, so that the QCD (pseudo)critical line and the freeze-out curves lie close to each other. In general they can be parametrized, at low baryon densities, by a lowest order Taylor expansion in the baryon chemical potential, as follows

$$
\frac{T\left(\mu_{B}\right)}{T_{c}(0)}=1-\kappa\left(\frac{\mu_{B}}{T\left(\mu_{B}\right)}\right)^{2}
$$

where $T_{c}(0)$ is the (pseudo)critical temperature at vanishing baryon density.

The QCD (pseudo)critical line can be determined within a first-principle approach exploiting lattice gauge theory methods. But at nonzero baryon density, due to the well known "sign problem", the QCD fermion determinant becomes complex and standard numerical simulations are unfeasible. Several methods have been invented to attack this problem (for a review, see [4-7]). In the present work we exploit the method of analytic continuation from an imaginary chemical potential to give a first estimate of the QCD (pseudo)critical line using the HISQ/tree action [8,9] with $2+1$ staggered fermions (see also Ref. [10]). The strange mass is set at the physical value and simulations are performed on the line of constant physics (LCP) with the light quark mass fixed at $m_{l}=m_{s} / 20$, as determined in Ref. [1]. As for quark chemical potentials, in the present study we assign the same value to the three quark species, $\mu_{l}=\mu_{s} \equiv \mu$. We explore lattices of different spatial extensions, $16^{3} \times 6$ and $24^{3} \times 6$, to check for finite size effects, and present results on a $32^{3} \times 8$ lattice, to check for finite cut-off effects. 


\section{Numerical results and discussion}

We perform simulations of lattice QCD with 2+1 flavors of rooted staggered quarks at imaginary quark chemical potential. We have made use of the HISQ/tree action $[8,9]$ as implemented in the publicly available MILC code, suitably modified by us in order to introduce an imaginary quark chemical potential $\mu=\mu_{B} / 3$. All simulations make use of the rational hybrid Monte Carlo (RHMC) algorithm. The length of each RHMC trajectory has been set to 1.0 in molecular dynamics time units. Simulations have been done on lattices of size $16^{3} \times 6,24^{3} \times 6$ and $32^{3} \times 8$. We have discarded typically not less than one thousand trajectories for each run and have collected from $4 \mathrm{k}$ to $8 \mathrm{k}$ trajectories for each measurement. The (pseudo)critical line $\beta_{c}\left(\mu^{2}\right)$ has been determined as the value for which the disconnected susceptibility of the light quark chiral condensate exhibits a peak. To precisely localize the peak, a Lorentzian fit has been used. Since we want to determine the ratio $T_{c}(\mu) / T_{c}(0)$, we need to set the lattice spacing. This can be done following the discussion in Appendix B of Ref. [1], where, for this particular value of $m_{l} / m_{s}$, the spacing is given in terms of the $r_{1}$ parameter:

$$
\frac{a}{r_{1}}(\beta)_{m_{l}=0.05 m_{s}}=\frac{c_{0} f(\beta)+c_{2}(10 / \beta) f^{3}(\beta)}{1+d_{2}(10 / \beta) f^{2}(\beta)},
$$

with $c_{0}=44.06, c_{2}=272102, d_{2}=4281, r_{1}=0.3106(20) \mathrm{fm} \mathrm{[11]} \mathrm{and}$

$$
f(\beta)=\left(b_{0}(10 / \beta)\right)^{-b_{1} /\left(2 b_{0}^{2}\right)} \exp \left(-\beta /\left(20 b_{0}\right)\right),
$$

where $b_{0}$ and $b_{1}$ are the universal coefficients of the two-loop beta function. From $a(\beta)$ we determine, for each explored lattice size separately, $T_{c}(\mu) / T_{c}(0)=a\left(\beta_{c}(0)\right) / a\left(\beta_{c}(\mu)\right)$. Data for $T_{c}(\mu) / T_{c}(0)$ versus $\mu /(\pi T)$ are reported in Fig. 1 . For the $16^{3} \times 6$ lattice, where the determination at three different values of $\mu$ is available, we have tried a linear fit in $\mu^{2}$ :

$$
\frac{T_{c}(\mu)}{T_{c}(0)}=1+R_{q}\left(\frac{i \mu}{\pi T_{c}(\mu)}\right)^{2},
$$

which works well over the whole explored range $\left(\chi^{2} /\right.$ d.o.f. $\left.=0.39\right)$ and gives us access to the curvature $R_{q}$. On the other lattices, assuming that linearity in $\mu^{2}$ still holds, we can extract $R_{q}$ from the determination at $\mu /(\pi T)=0.2 i$; we notice that such an assumption, for the given value of $\mu$, is consistent with all previous studies on the systematics of analytic continuation [16-19]. Our results are:

$$
\begin{array}{ll}
R_{q}\left(16^{3} \times 6\right)=-1.63(22), & \kappa=0.0183(24), \\
R_{q}\left(24^{3} \times 6\right)=-1.51(25), & \kappa=0.0170(28), \\
R_{q}\left(32^{3} \times 8\right)=-1.70(29), & \kappa=0.0190(32),
\end{array}
$$

where $\kappa=-R_{q} /\left(9 \pi^{2}\right)$ is the curvature parameter introduced in Eq. (1.1). The results provide evidence that finite size and finite cut-off systematic effects are within our present statistical uncertainties. We cannot yet try an extrapolation to the continuum limit of our results, however, taking into account the statistical errors and the observed variations of the results with the lattice size and the ultraviolet cutoff, our present estimate for kappa is

$$
\kappa=0.018(4) \text {. }
$$




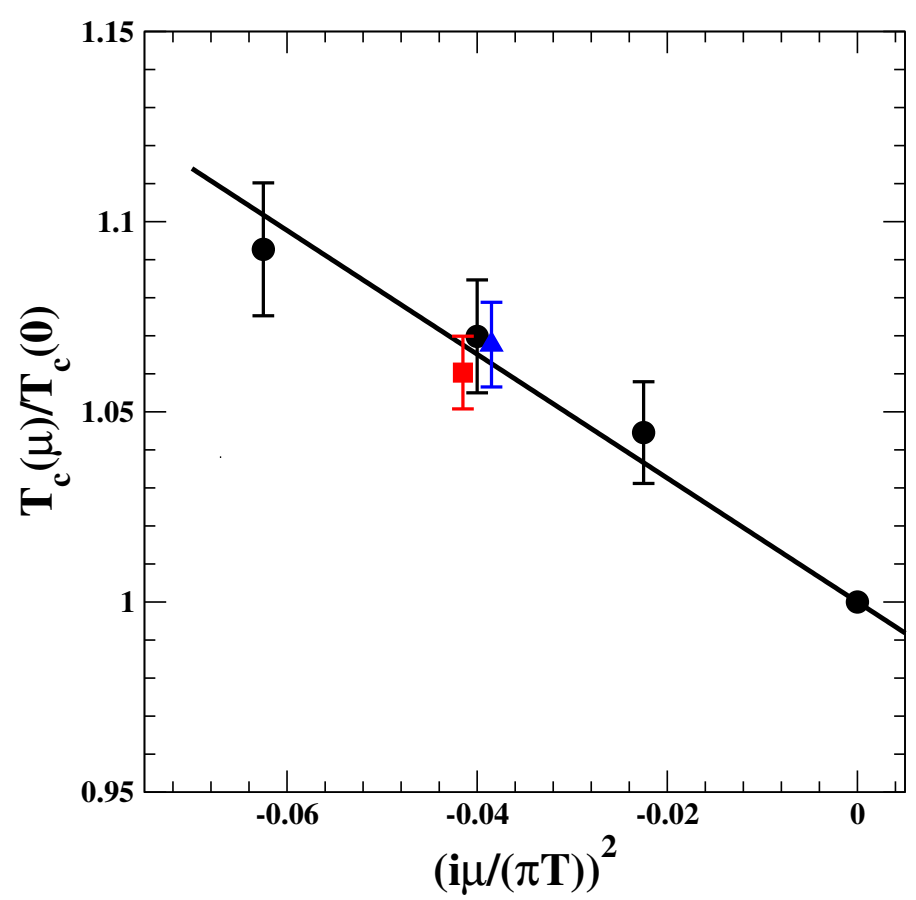

Figure 1: $T_{c}(\mu) / T_{c}(0)$ versus $((i \mu) /(\pi T))^{2}$ obtained on a $16^{3} \times 6$ lattice (full circles), on a $24^{3} \times 6$ lattice (full square) and on a $32^{3} \times 8$ lattice (full triangle). For the sake of readability the abscissae at $((i \mu) /(\pi T))^{2}=-0.04$ for $24^{3} \times 6$ and $32^{3} \times 8$ data have been slightly shifted. The full line is a linear fit to the data on the $16^{3} \times 6$ lattice.

In Figure 2 we compare our determination of the curvature $\kappa$ defined in Eq. (1.1) with other lattice results and with the estimates of the freeze-out curve. A preliminary result from the Pisa group [12], obtained using the analytic continuation, gives $\kappa=0.0153(14)$ from the renormalized chiral susceptibility and $\kappa=0.0144(7)$ from the renormalized chiral condensate on a $32^{2} \times 8$ lattice. The Budapest-Wuppertal collaboration [14], using a Symanzik improved gauge action and stout-link improved staggered fermions on lattices with temporal size $N_{t}=6,8,10$ and aspect ratios equal to three and four, finds, after continuum extrapolation, $\kappa=0.0089(14)$ by the Taylor expansion method with the strange quark number susceptibility as probe observable and $\kappa=0.0066(20)$ when, instead, the renormalized chiral condensate is used. The Bielefeld-BNL collaboration [13], using the p4-action on lattices with $N_{t}=4$ and 8 , and aspect ratios up to four, finds $\kappa=0.0066(7)$ again with the Taylor expansion method and the light quark susceptibility as a probe observable. Another collaboration [15] adopted improved staggered fermions in the p4fat 3 version, on lattices with $N_{t}=4$ and aspect ratio four with physical strange quark mass and pion mass at $220 \mathrm{MeV}$, getting $\kappa=0.0100$ (2) by the method of analytic continuation, with the Polyakov loop phase as a probe. Regarding the freeze-out curve, we report two different estimates. The first is from the analysis of Ref. [2], which is based on the standard statistical hadronization model; there the authors parametrize the freeze-out curve as

$$
T_{c}\left(\mu_{B}\right)=a-b \mu_{B}^{2}-c \mu_{B}^{4}
$$

with $a=0.166(2) \mathrm{GeV}, b=0.139(16) \mathrm{GeV}^{-1}$, and $c=0.053(21) \mathrm{GeV}^{-3}$, from which we have 


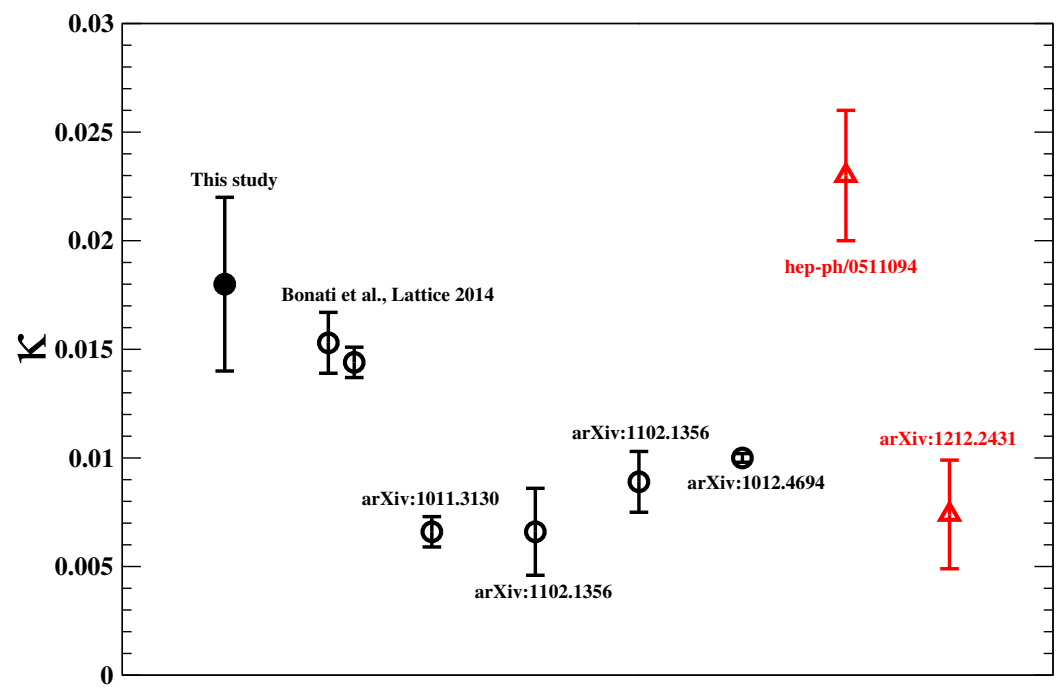

Figure 2: Comparison of different determinations of the curvature of the chemical freeze-out curve and of the (pseudo)critical line for QCD with $n_{f}=2+1$. From left to right: $i$ ) analytic continuation, disconnected chiral susceptibility, this study; ii) analytic continuation, renormalized chiral susceptibility, Ref. [12]; iii) analytic continuation, renormalized chiral condensate, Ref. [12]; iv) Taylor expansion, chiral susceptibility, Ref. [13]; v) Taylor expansion, chiral condensate, Ref. [14]; vi) Taylor expansion, strange quark number susceptibility, Ref. [14]; vii) analytic continuation, Polyakov loop, Ref. [15]; viii) freeze-out curvature, standard analysis, Ref. [2]; ix) freeze-out curvature, revised analysis of Ref. [3].

derived the $\kappa$ value reported in Fig.2. The second estimate is based on the freeze-out points which are reported in Table I of Ref. [3] and are based on a modified statistical reanalysis of the experimental data which includes the effects of inelastic collisions taking place after freeze-out. Our result for the curvature is typically between two and three standard deviations larger than previous lattice determinations and seems in a better agreement with the freeze-out curvature based on the standard statistical hadronization model. Possible reasons for the disagreement with previous lattice determinations can lie in the different methods adopted to avoid the sign problem, in the different lattice discretizations, as well as in the different observables used to locate the transition point, and in the setup of quark chemical potentials. We also report, in Fig. 3, an estimate of the (pseudo)critical line which is based on our determination of the curvature. Regarding the value of $T_{c}$ at $\mu_{B}=0$, which is affected by larger finite size and finite cutoff effects than $\kappa$, we refer directly to the presently accepted continuum extrapolated value, $T_{c} \sim 155$ [1,20-22], and in particular to the one obtained in Ref. [1] with the same action adopted in our study, $T_{c}(0)=154(9) \mathrm{MeV}$. From that and from $\kappa=0.018(4)$ we obtain $b=0.117(27) \mathrm{GeV}^{-1}$ (see Eq. 2.6). Freeze-out determinations from Refs. [2,3] are reported as well.

Let us conclude by discussing the possible sources of systematic effects in our estimate. One of them is related to the extrapolation from imaginary to real chemical potentials: in the case of the $16^{3} \times 6$ lattice we have performed simulations at different values of imaginary $\mu$, thus verifying that a linear interpolation (in $\mu^{2}$ ) of data works well. For the other two lattices, instead, we have considered only one value of imaginary $\mu(\mu /(\pi T)=0.2 i)$ and the linear behavior has been assumed. Our previous studies based on analytic continuation, however, indicate that the chosen 


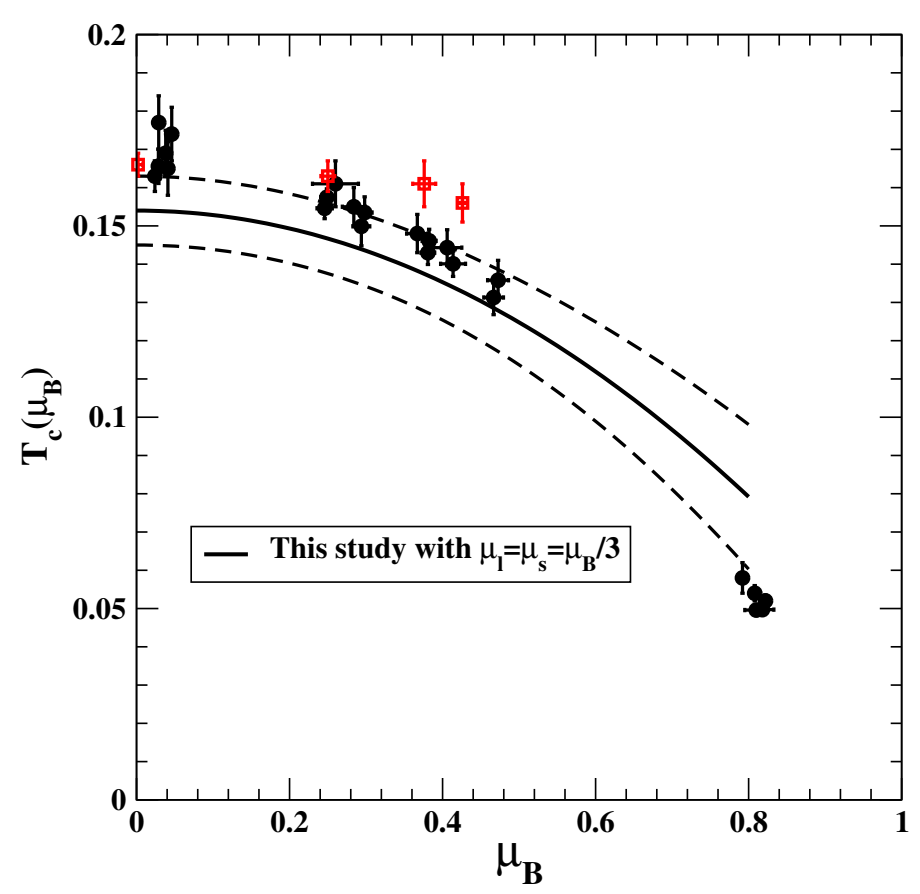

Figure 3: $T_{c}\left(\mu_{B}\right)$ versus $\mu_{B}$ (units in $\left.\mathrm{GeV}\right)$. Experimental values of $T_{c}\left(\mu_{B}\right)$ are taken from Fig. 1 of Ref. [2] (circles) and from Table I of Ref. [3] (squares) respectively for the standard and the modified statistical hadronization model. The solid line is a parametrization corresponding to $T_{c}\left(\mu_{B}\right)=T_{c}(0)-b \mu_{B}^{2}$ with $T_{c}(0)=$ $0.154(9) \mathrm{GeV}$ and $b=0.117(27) \mathrm{GeV}^{-1}$. The dashed lines represent the corresponding error band.

value of $\mu$ should lie well inside the region of linearity. Nevertheless, we plan to perform a more systematic study of this issue. Finally, we have verified that finite size and cutoff effects are under control, within the present statistical accuracy. Still, the extrapolation to the continuum limit, as well as the extension to the physical value of the light to strange mass ratio, $m_{l} / m_{s} \sim 1 / 28$, and the possible effect of varying the strange quark chemical potential, deserve further investigations and will be the subject of forthcoming works.

\section{Acknowledgements}

This work was based in part on the MILC Collaboration's public lattice gauge theory code (http://physics.utah.edu/ detar/milc.html) and has been partially supported by INFN SUMA project. Simulations have been performed on BlueGene/Q at CINECA (CINECAINFN agreement).

\section{References}

[1] A. Bazavov, T. Bhattacharya, M. Cheng, C. DeTar, H. Ding, et. al., The chiral and deconfinement aspects of the QCD transition, Phys.Rev. D85 (2012) 054503, [arXiv:1111.1710].

[2] J. Cleymans, H. Oeschler, K. Redlich, and S. Wheaton, Comparison of chemical freeze-out criteria in heavy-ion collisions, Phys.Rev. C73 (2006) 034905, [hep-ph / 0511094 ]. 
[3] F. Becattini, M. Bleicher, T. Kollegger, T. Schuster, J. Steinheimer, et. al., Hadron Formation in Relativistic Nuclear Collisions and the QCD Phase Diagram, Phys.Rev.Lett. 111 (2013) 082302, [arXiv:1212.2431].

[4] O. Philipsen, The QCD phase diagram at zero and small baryon density, PoS LAT2005 (2006) 016, [hep-lat/0510077].

[5] C. Schmidt, Lattice QCD at finite density, PoS LAT2006 (2006) 021, [hep-lat/ 0610116 ].

[6] P. de Forcrand, Simulating QCD at finite density, PoS LAT2009 (2009) 010, [arXiv: 1005.0539 ].

[7] G. Aarts, Complex Langevin dynamics and other approaches at finite chemical potential, PoS LATTICE2012 (2012) 017, [arXiv:1302.3028].

[8] E. Follana et. al., Highly improved staggered quarks on the lattice, with applications to charm physics, Phys.Rev. D75 (2007) 054502, [hep-lat/ 0610092$].$

[9] A. Bazavov et. al., Scaling studies of QCD with the dynamical HISQ action, Phys.Rev. D82 (2010) 074501, [arXiv: 1004.0342$]$.

[10] P. Cea, L. Cosmai, and A. Papa, Critical line of 2+1 flavor QCD, Phys.Rev. D89 (2014) 074512, [arXiv:1403.0821].

[11] A. Bazavov et. al., Results for light pseudoscalar mesons, PoS LATTICE2010 (2010) 074, [arXiv:1012.0868].

[12] C. Bonati, M. D'Elia, M. Mariti, M. Mesiti, F. Negro, and F. Sanfilippo, The curvature of the $Q C D$ critical line from analytic continuation, in these Proceedings, 2014.

[13] O. Kaczmarek, F. Karsch, E. Laermann, C. Miao, S. Mukherjee, et. al., Phase boundary for the chiral transition in (2+1) -flavor QCD at small values of the chemical potential, Phys.Rev. D83 (2011) 014504, [arXiv:1011.3130].

[14] G. Endrodi, Z. Fodor, S. Katz, and K. Szabo, The QCD phase diagram at nonzero quark density, JHEP 1104 (2011) 001, [arXiv:1102.1356].

[15] R. Falcone, E. Laermann, and M. P. Lombardo, Study of finite temperature QCD with 2+1 flavors via Taylor expansion and imaginary chemical potential, PoS LATTICE2010 (2010) 183, [arXiv:1012.4694].

[16] P. Cea, L. Cosmai, M. D'Elia, and A. Papa, The phase diagram of QCD with four degenerate quarks, Phys.Rev. D81 (2010) 094502, [arXiv: 1004 .0184].

[17] P. Cea, L. Cosmai, M. D'Elia, and A. Papa, The critical line of QCD with four degenerate quarks, PoS LATTICE2010 (2010) 173, [arXiv: 1012 .4908].

[18] P. Cea, L. Cosmai, M. D’Elia, A. Papa, and F. Sanfilippo, Two-flavor QCD at finite quark or isospin density, PoS LATTICE2012 (2012) 067, [arXiv: 1210 . 5896 ].

[19] P. Cea, L. Cosmai, M. D’Elia, A. Papa, and F. Sanfilippo, The critical line of two-flavor QCD at finite isospin or baryon densities from imaginary chemical potentials, Phys.Rev. D85 (2012) 094512, [arXiv:1202.5700].

[20] Y. Aoki, Z. Fodor, S. Katz, and K. Szabo, The QCD transition temperature: Results with physical masses in the continuum limit, Phys.Lett. B643 (2006) 46-54, [hep-lat / 0609068 ].

[21] Y. Aoki, S. Borsanyi, S. Durr, Z. Fodor, S. D. Katz, et. al., The QCD transition temperature: results with physical masses in the continuum limit II., JHEP 0906 (2009) 088, [arXiv: 0903.4155$].$

[22] S. Borsanyi et. al., Is there still any $T_{c}$ mystery in lattice QCD? Results with physical masses in the continuum limit III, JHEP 1009 (2010) 073, [arXiv : 1005 . 3508 ]. 